Review began 09/24/2021 Review ended 09/26/2021 Published 09/29/2021

(c) Copyright 2021

Tze-Suen et al. This is an open access article distributed under the terms of the Creative Commons Attribution License CCBY 4.0., which permits unrestricted use, distribution, and reproduction in any medium, provided the original author and source are credited.

\section{Fungal Keratitis in a Tertiary Hospital in Malaysia}

\author{
Chow Tze-Suen ${ }^{1}$, Tan Chew-Ean ${ }^{1}$, Norshamsiah Md Din ${ }^{2}$
}

1. Department of Ophthalmology, Hospital Sultanah Bahiyah, Alor Setar, MYS 2. Department of Ophthalmology, Universiti Kebangsaan Malaysia Medical Centre, Kuala Lumpur, MYS

Corresponding author: Chow Tze-Suen, chowtzesuen@gmail.com

\section{Abstract \\ Background}

Fungal keratitis is one of the commonest causes of corneal blindness in developing countries including Malaysia. We aim to evaluate the sociodemographic background, clinical features, predisposing factors, laboratory findings, management, complications, and visual outcome of patients with fungal keratitis in a tertiary hospital in Malaysia.

\section{Methods}

A retrospective review of medical and microbiology records of all patients treated for corneal ulcer from 2015 to 2019 at Hospital Sultanah Bahiyah was performed. Sociodemography, predisposing risk factors, clinical characteristics, causative organisms, and final visual outcome were analyzed.

\section{Results}

We identified 103 patients with a diagnosis of fungal keratitis. The majority of the patients were of Malay ethnicity (85.4\%) and male gender (81.6\%), with an age range of 21 to 60 years $(94.1 \%)$. Ocular trauma was the main predisposing factor in 82 (79.6\%) patients. Poor visual improvement was observed in groups with ulcer more than $4 \mathrm{~mm}$ (67.5\%), presence of hypopyon (50.9\%), and high intraocular pressure (75.0\%) upon presentation. Fusarium spp. (19.4\%) was the commonest fungus isolated followed by Aspergillus spp. (5.8\%). All patients were prescribed either topical, oral, intracameral, or combined therapy, whereas 20 (19.4\%) patients required surgical intervention, of which $16(15.5 \%)$ underwent penetrating keratoplasty and three (2.9\%) required evisceration.

\section{Conclusion}

The epidemiological, socioeconomic, and predisposing factors may facilitate timely diagnosis and prompt treatment to achieve a better visual outcome and minimize complications including corneal blindness.

Categories: Ophthalmology

Keywords: fungus, evisceration, penetrating keratoplasty, fungal keratitis, corneal ulcer

\section{Introduction}

Fungal keratitis is one of the commonest causes of corneal blindness, contributing to $24.8 \%$ of all corneal ulcer cases in Malaysia [1]. The World Health Organization (WHO) found out that $4 \%$ cases of all blindness are caused by corneal opacities [1]. Several predisposing factors were identified, including ocular trauma, prolonged use of topical steroid, underlying ocular surface disease, contact lens wear, diabetes mellitus, and immunosuppression. Malaysia with its warm climate and high humidity year-round provides a conducive environment for sporadic fungal growth. Hence, high clinical suspicion is vital even if there is no significant history of trauma by a vegetative matter or direct inoculation $[2,3]$.

Clinical manifestations supported by corneal scraping microscopy examination and culture remain the mainstay of diagnosis. Fusarium spp., Candida spp., and Aspergillus spp. are frequently found in the cultures from corneal scraping [4]. Because of the potential delay in culture confirmation, early initiation of antifungal treatment should be based on clinical judgment to achieve a better outcome and minimize devastating complications such as corneal scarring, corneal perforation, and endophthalmitis $[2,4]$.

We aim to report the sociodemographic background, clinical features, predisposing factors, laboratory findings, management, complications, and visual outcome of patients in a tertiary hospital in Malaysia. This information will allow the profiling of fungal ulcers and facilitate early diagnosis and prompt treatment in future cases.

\section{Materials And Methods}

This is a retrospective review of patients diagnosed with fungal keratitis in Hospital Sultanah Bahiyah, a 
tertiary referral center in northern Malaysia. This study was granted ethical approval by the Malaysian National Institute of Health and was registered in the National Medical Research Registry (NMRR-20-1473$55317)$.

All corneal scrape samples undertaken from January 2015 to December 2019 were extracted from electronic records. Patients with the diagnosis of fungal keratitis were identified and included in this study. The database was then used to retrieve patients' demographic information, clinical comorbidities, predisposing factors, clinical features of fungal keratitis, laboratory findings, duration of hospitalization, treatments, complications, and visual outcome of the patients.

The comprehensive assessment included medical history, presenting visual acuity, slit-lamp biomicroscopic examination, and corneal scraping of the affected eye. Microscopic evaluation, which included gram stain and $10 \%$ potassium hydroxide $(\mathrm{KOH})$ mount, was performed on collected corneal smears as part of the standard protocol. Other culture samples were sent using blood agar, chocolate agar, Sabouraud dextrose agar, and MacConkey agar.

Treatment given to patients was individualized based on clinical evaluation and laboratory findings. Routine follow-ups at regular intervals were conducted to assess the treatment efficacy, disease progression, and possible complications. Final visual acuity was taken at least six months after completion of treatment. Visual acuity was recorded using the Snellen chart, grouped as good (6/6 to 6/12), moderate (6/15 to 6/60), and severe (worse than 3/60) [5]. Patients with incomplete data were excluded from this study.

Statistical analysis for descriptive analysis was performed using SPSS 22.0 (IBM Corp., Armonk, NY). Pearson's chi-square test was used to determine the relationship between two categorical data. A p-value of less than 0.05 was considered statistically significant.

\section{Results}

A total of 103 patients with the diagnosis of fungal keratitis were enrolled in this study. The mean age was $51.1 \pm 17.1$ years (range: $19-87$ years). The majority of patients were male $(n=84,81.6 \%)$, while $19(18.4 \%)$ were female. Majority of them Malay ethnicity (85.4\%), followed by Chinese (6.8\%), non-Malaysian (6.8\%), and Indian (1.0\%). Only 15 (14.6\%) smokers were recorded, while 17 (16.5\%) patients had diabetes mellitus (Table 1). 


\section{Cureus}

\begin{tabular}{|c|c|c|}
\hline Demographic variables & Frequency $(\mathbf{n})$ & Percentage (\%) \\
\hline \multicolumn{3}{|l|}{ Age (years) } \\
\hline$<21$ & 1 & 1.0 \\
\hline $21-40$ & 37 & 35.9 \\
\hline $41-60$ & 29 & 28.2 \\
\hline $61-80$ & 31 & 30.1 \\
\hline$>80$ & 5 & 4.9 \\
\hline \multicolumn{3}{|l|}{ Gender } \\
\hline Male & 84 & 81.6 \\
\hline Female & 19 & 18.4 \\
\hline \multicolumn{3}{|l|}{ Ethnicity } \\
\hline Malay & 88 & 85.4 \\
\hline Chinese & 7 & 6.8 \\
\hline Indian & 1 & 1.0 \\
\hline Others/foreigners & 7 & 6.8 \\
\hline \multicolumn{3}{|l|}{ Smoker } \\
\hline Yes & 15 & 14.6 \\
\hline No & 88 & 85.4 \\
\hline \multicolumn{3}{|l|}{ Diabetes mellitus } \\
\hline Yes & 17 & 16.5 \\
\hline No & 86 & 83.5 \\
\hline
\end{tabular}

TABLE 1: Sociodemographics of patients

Our findings showed that ocular trauma was the major predisposing ocular factor $(82,79.6 \%)$, followed by corneal suture related in six (5.8\%) patients, contact lens related in four (3.9\%) patients, bullous keratopathy in three (2.9\%) patients, ocular surface disease in two (1.9\%) patients, and no known predisposing factor in six (5.8\%) patients. Among those with ocular trauma, $29.1 \%$ were due to vegetative matter as compared to 27 (26.2\%) cases caused by non-vegetative matter such as stone, sand, dust, metal, chemical, and insect (Table 2). 


\section{Cureus}

\begin{tabular}{|c|c|c|}
\hline Predisposing ocular factors & Frequency (n) & Percentage (\%) \\
\hline Ocular trauma & 82 & 79.6 \\
\hline Vegetative & 30 & 29.1 \\
\hline Non-vegetative & 27 & 26.2 \\
\hline Unknown & 25 & 24.3 \\
\hline Ocular surface disease & 2 & 1.9 \\
\hline Contact lens related & 4 & 3.9 \\
\hline Bullous keratopathy & 3 & 2.9 \\
\hline Corneal suture related & 6 & 5.8 \\
\hline None & 6 & 5.8 \\
\hline
\end{tabular}

TABLE 2: Predisposing ocular factors for fungal keratitis

Table 3 showed the final visual acuity in our patients in relation to various clinical features. Large ulcer diameter $(p<0.001)$, central location of the fungal ulcer $(p=0.006)$, presence of hypopyon $(p=0.002)$, and high intraocular pressure (IOP) $(p<0.001)$ were significantly associated with worse final visual acuity. The duration of symptoms prior to presentation had no important association with visual outcome $(\mathrm{p}>0.05)$ (Table 3). 


\section{Cureus}

\begin{tabular}{|c|c|c|c|c|}
\hline & \multicolumn{3}{|c|}{ Final visual acuity*, n (\%) } & \multirow[t]{2}{*}{ p-Value } \\
\hline & Good & Moderate & Severe & \\
\hline \multicolumn{5}{|c|}{ Presenting interval (days) } \\
\hline$\leq 3$ & $23(42.6)$ & $11(20.4)$ & $20(37.0)$ & \multirow{2}{*}{0.114} \\
\hline$>3$ & $13(26.5)$ & $18(36.7)$ & $18(36.7)$ & \\
\hline \multicolumn{5}{|c|}{ Size, largest diameter (mm) } \\
\hline$\leq 2$ & $17(65.4)$ & $4(15.4)$ & $5(19.2)$ & \multirow{3}{*}{$<0.001$} \\
\hline $2-4$ & $16(43.2)$ & $15(40.5)$ & $6(16.2)$ & \\
\hline$>4$ & $3(7.5)$ & $10(25.0)$ & $27(67.5)$ & \\
\hline \multicolumn{5}{|l|}{ Location } \\
\hline Central & $5(15.6)$ & $7(21.9)$ & $20(62.5)$ & \multirow{3}{*}{0.006} \\
\hline Paracentral & $24(43.6)$ & $18(32.7)$ & $13(23.6)$ & \\
\hline Peripheral & $7(43.8)$ & $4(25.0)$ & $5(31.3)$ & \\
\hline \multicolumn{5}{|l|}{ Hypopyon } \\
\hline Present & $13(22.8)$ & $15(26.3)$ & $29(50.9)$ & \multirow[t]{2}{*}{0.002} \\
\hline Absent & $23(50.0)$ & $14(30.4)$ & $9(19.6)$ & \\
\hline \multicolumn{5}{|c|}{ Intraocular pressure (mm Hg) } \\
\hline $10-21$ & $36(43.3)$ & $24(28.9)$ & $23(27.7)$ & \multirow{2}{*}{$<0.001$} \\
\hline$>21$ & $0(0.0)$ & $5(25.0)$ & $15(75.0)$ & \\
\hline
\end{tabular}

TABLE 3: Presenting and final visual acuity based on clinical features

*Visual acuity: good, 6/6-6/12; moderate, 6/15-3/60; severe, $<3 / 60$

${ }^{\dagger}$ Pearson's chi-square test: $p<0.05$ is significant

Out of 103 cases, causative fungi were successfully isolated in 41 (39.8\%) cases, in which Fusarium spp. was mostly yielded (20, 19.4\%) followed by Aspergillus spp. (n=6, 5.8\%) (Table 4). 


\section{Cureus}

\begin{tabular}{|c|c|c|}
\hline & Frequency $(\mathbf{n})$ & Percentage $(\%)$ \\
\hline \multicolumn{3}{|l|}{ Causative fungus } \\
\hline Fusarium spp. & 20 & 19.4 \\
\hline Aspergillus spp. & 6 & 5.8 \\
\hline Curvularia spp. & 3 & 2.9 \\
\hline Non-sporulating hyaline hyphomycetes & 3 & 2.9 \\
\hline Candida spp. & 2 & 1.9 \\
\hline Bipolaris spp. & 2 & 1.9 \\
\hline Nigrospora spp. & 2 & 1.9 \\
\hline Chrysosporium spp. & 1 & 1.0 \\
\hline Unidentified & 62 & 60.2 \\
\hline \multicolumn{3}{|l|}{ Antifungal prescribed } \\
\hline Single topical antifungal & 9 & 8.7 \\
\hline Dual topical antifungals & 40 & 38.8 \\
\hline Combined topical and oral antifungals & 44 & 42.7 \\
\hline Topical, oral, and intracameral antifungals & 10 & 9.7 \\
\hline \multicolumn{3}{|l|}{ Surgical intervention } \\
\hline Penetrating keratoplasty & 16 & 15.5 \\
\hline Evisceration & 3 & 2.9 \\
\hline Corneal glue with bandage contact lens & 3 & 2.9 \\
\hline Tarsorrhaphy & 1 & 1.0 \\
\hline
\end{tabular}

TABLE 4: Causative fungus, antifungal prescribed, and surgical Intervention

The treatment regime was decided based on clinical manifestation, progression, and response to treatment. Most $(44,42.7 \%)$ patients were treated with combined topical and oral antifungals, followed by dual topical antifungals (40,38.8\%), combined topical, oral, and intracameral antifungals (10,9.7\%), and single topical antifungal (9, 8.7\%) (Table 4).

A total of 20 (19\%) patients had to undergo surgical intervention including penetrating keratoplasty (PK) $(n=16,15.5 \%)$, evisceration $(n=3,2.9 \%)$, corneal glue with bandage contact lens $(n=3,2.9 \%)$, and tarsorrhaphy ( $\mathrm{n}=1,1.0 \%)$ (Table 4).

\section{Discussion}

Fungal keratitis is a great public health threat especially in developing countries as it is one of the major causes of corneal blindness in a relatively younger age group $[4,6]$. The annual global incidence of fungal keratitis is 23.6 per 100,000 population but this figure is particularly higher in Asia mainly in the southern and south-eastern regions, with an incidence of 73 and 15 cases per 100,000 population, respectively $[4,6]$. This can be attributed to the warm weather and high humidity, as well as agricultural and outdoor work as main economic activities in these regions [6]. Our study recorded an annual incidence of five cases per 100,000 population, which is higher than Malaysia's national average incidence of 1.3 per 100,000 population [6].

Kedah is a state in northern Malaysia where agriculture is the main economic activity. All these geographic and socioeconomic properties are the main contributing factors to a higher incidence of fungal keratitis in our population. Local studies in other states of Malaysia with a similar background supported the findings in our study $[3,4,5,7]$. Besides that, we also discover the relevance of the abovementioned factors in the higher incidence of fungal keratitis in India and China $[1,5,8,9]$. 
The patient profile in our study found that most of them were Malay (85\%), male (82\%), and young (65\% aged 21 to 60 years). Ocular trauma resulted in almost $80 \%$ of cases, predominantly caused by vegetative matters $(30,29.1 \%)$. This conforms with the local sociodemographic profile in which Malay is the major ethnicity and young males are the main workforce in a largely agricultural state, including farmers, fishermen, and laborers. Our results are in line with several other local studies $[1,2,5]$. Koh et al. found that diabetes mellitus was a common risk factor for corneal ulcer due to impaired corneal barrier function and delayed epithelial healing [10]. However, no significant relationship can be established between smokers or diabetic patients with fungal keratitis in our study.

Visual acuity was assessed using the Snellen chart at the first presentation and subsequent visits. Final visual acuity was assessed at least six months from the completion of optimal treatment. Delayed presentation and treatment are known to be associated with poorer visual outcome. Around $47.5 \%$ of patients in our study presented more than three days from the onset. Factors contributing to the delayed presentation included lack of awareness, self-remedy, and over-the-counter drugs used. Our study demonstrated that large ulcer diameter $(>4 \mathrm{~mm})$, central fungal ulceration, presence of hypopyon, and high IOP (> $21 \mathrm{mmHg}$ ) on presentation were poor prognostic factors for the final visual outcome. Our study findings were parallel with studies conducted by Chitamparam et al. and Prajna et al. [7,11]. Central fungal ulceration disrupted the visual axis, resulting in worse final vision as compared to paracentral and peripheral ulceration [7].

Fusarium spp., Aspergillus spp., and Candida spp. are known to be the commonest causative fungi in mycotic keratitis [6]. Our study revealed Fusarium spp. was the main causative fungus (19.4\%) followed by Aspergillus spp. (5.8\%). Yap et al., Mohd-Tahir et al., and Chitamparam et al. also demonstrated Fusarium spp. as the commonest causative organism in fungal keratitis $[1,2,7]$.

Most of our patients received either dual topical antifungal (amphotericin B and fluconazole) or combined topical and oral antifungal (fluconazole). Only $10 \%$ of our patients required combined topical, oral, and intracameral antifungal. Cochrane review suggested that natamycin was superior in treating Fusarium fungal ulcers compared to other antifungal agents. However, natamycin is expensive and is not routinely available in Malaysia due to its cost [12]. Sharma et al. showed that there was no significant extra benefit of intracameral amphotericin B over topical therapy in the Indian population [13].

Surgical treatment is indicated in cases refractory to medical therapy and deep or severe fungal keratitis [14]. Twenty (19.4\%) patients from our study had to undergo surgical intervention such as PK and evisceration. The surgical rate in our study is almost similar to another local study in Kelantan by MohdTahir et al. who showed that $19.5 \%$ of patients required PK and $4.3 \%$ required evisceration [2]. A study carried out in North China recorded $37.8 \%$ of PK was performed in their patients due to delay in presentation and wrong usage of drugs received prior to admission [14]. PK remains the commonest surgical intervention with promising improvement in visual outcome $[15,16]$.

There are several limitations to our study. Due to the retrospective nature and incomplete medical records, sociodemographic information such as occupation, education level, and household income could not be evaluated. Efficacy of different treatment regime is also incomparable due to the retrospective nature of this study and the lack of standardized treatment protocol. In any case, the existing data may help identify commoner causative organisms based on the clinical features and predict the patients' final visual outcome.

\section{Conclusions}

Ocular trauma was the main predisposing factor in our study due to high involvement in agricultural and outdoor activities. Bigger ulcer size, central corneal involvement, presence of hypopyon, high IOP, and poor vision at presentation are factors for poorer final visual outcome. A high index of suspicion of fungal keratitis especially when treating patients with known predisposing factors facilitates early diagnosis and prompt treatment, preventing unfavorable visual outcome.

\section{Additional Information}

\section{Disclosures}

Human subjects: Consent was obtained or waived by all participants in this study. Malaysian National Institute of Health issued approval NMRR-20-1473-55317. Animal subjects: All authors have confirmed that this study did not involve animal subjects or tissue. Conflicts of interest: In compliance with the ICMJE uniform disclosure form, all authors declare the following: Payment/services info: All authors have declared that no financial support was received from any organization for the submitted work. Financial relationships: All authors have declared that they have no financial relationships at present or within the previous three years with any organizations that might have an interest in the submitted work. Other relationships: All authors have declared that there are no other relationships or activities that could appear to have influenced the submitted work.

\section{References}


1. Yap JY, Tan KV, Sonny-Teo KH: A 3-year retrospective review of corneal ulcers in Hospital Univesiti Sains Malaysia. Fam Med Prim Care Rev. 2019, 21:62-6. 10.5114/fmpcr.2019.82980

2. Mohd-Tahir F, Norhayati A, Siti-Raihan I, Ibrahim M: A 5-year retrospective review of fungal keratitis at Hospital University Sains Malaysia. Interdiscip Perspect Infect Dis. 2012, 2012:851563.

$10.1155 / 2012 / 851563$

3. Gopinathan U, Garg P, Fernandes M, Sharma S, Athmanathan S, Rao GN: The epidemiological features and laboratory results of fungal keratitis: a 10-year review at a referral eye care center in South India. Cornea. 2002, 21:555-9. 10.1097/00003226-200208000-00004

4. Ansari Z, Miller D, Galor A: Current thoughts in fungal keratitis: diagnosis and treatment. Curr Fungal Infect Rep. 2013, 7:209-18. 10.1007/s12281-013-0150-110.1007/s12281-013-0150-1

5. Omar N Muna'aim MA, Saleh RM, Kasim ZM, Isa MM: An 8 year retrospective review if microbial keratitis in a secondary referral centre in Malaysia. Mal J Med Health Sci. 2017, 13:47-57.

6. Brown L, Leck AK, Gichangi M, Burton MJ, Denning DW: The global incidence and diagnosis of fungal keratitis. Lancet Infect Dis. 2021, 21:e49-57. 10.1016/S1473-3099(20)30448-5

7. Chitamparam S, Lim TH, Tai E, Ibrahim M: Mycotic keratitis in a tertiary hospital in northeastern Malaysia . Turk J Ophthalmol. 2020, 50:332-8. 10.4274/tjo.galenos.2020.57609

8. Rautaraya B, Sharma S, Kar S, Das S, Sahu SK: Diagnosis and treatment outcome of mycotic keratitis at a tertiary eye care center in eastern India. BMC Ophthalmol. 2011, 11:39. 10.1186/1471-2415-11-39

9. Saha S, Banerjee D, Khetan A, Sengupta J: Epidemiological profile of fungal keratitis in urban population of West Bengal, India. Oman J Ophthalmol. 2009, 2:114-8. 10.4103/0974-620X.57310

10. Koh YY, Sun CC, Hsiao CH: Epidemiology and the estimated burden of microbial keratitis on the health care system in Taiwan: A 14-year population-based study. Am J Ophthalmol. 2020, 220:152-9. 10.1016/j.ajo.2020.07.026

11. Prajna NV, Krishnan T, Rajaraman R, et al.: Predictors of corneal perforation or need for therapeutic keratoplasty in severe fungal keratitis: a secondary analysis of the Mycotic Ulcer Treatment Trial II. JAMA Ophthalmol. 2017, 135:987-91. 10.1001/jamaophthalmol.2017.2914

12. FlorCruz NV, Peczon IV, Evans JR: Medical interventions for fungal keratitis. Cochrane Database Syst Rev. 2012, CD004241. 10.1002/14651858.CD004241.pub3

13. Sharma N, Sankaran $P$, Agarwal T, et al.: Evaluation of intracameral amphotericin B in the management of fungal keratitis: randomized controlled trial. Ocul Immunol Inflamm. 2016, 24:493-7. 10.3109/09273948.2015.1057597

14. Xie L, Zhong W, Shi W, Sun S: Spectrum of fungal keratitis in north China. Ophthalmology. 2006, 113:19438. 10.1016/i.ophtha. 2006.05 .035

15. Xie L, Dong X, Shi W: Treatment of fungal keratitis by penetrating keratoplasty . Br J Ophthalmol. 2001, 85:1070-4. 10.1136/bjo.85.9.1070

16. Xie L, Zhai H, Shi W: Penetrating keratoplasty for corneal perforations in fungal keratitis . Cornea. 2007, 26:158-62. 10.1097/01.ico.0000248381.24519.0d 